American Journal of Applied Sciences 7 (8): 1129-1138, 2010

ISSN 1546-9239

(C) 2010 Science Publications

\title{
Increasing the Reliability of Fuzzy Inference System-Based Skin Detector
}

\author{
Alaa Y. Taqa and Hamid A. Jalab \\ Department of Computer System and Technology, University of Malaya, \\ 50603, Kuala Lumpur, Malaysia
}

\begin{abstract}
Problem statement: Skin detection is a common primitive for many human-related image processing applications, such as video surveillance, naked image filters and face detection. Skin color is considered as a useful and discriminating spatial feature for many applications, but it is not robust enough to deal with complex image environments. Skin tones range from dark (some Africans) to light white (Caucasians and some Europeans). In addition, both the light-changing conditions and the existence of objects with skin-like colors could cause some major difficulties faced pixel-based skin detector depending only on a color feature. Approach: This study proposed a novel Fuzzy Inference System (FIS) for skin detection, which combines both color and texture features. To increase the reliability of the skin detection process, neighborhood pixel information is incorporated into the proposed method. The color feature is represented using RGB color model, while the texture feature is estimated using three statistical measures: standard deviation, entropy and range. The subtractive clustering-based fuzzy system method and the Sugeno type reasoning mechanism are used for modeling FIS-based skin detection. The proposed approach builds a fuzzy model of skin detection from existing images within skin and non-skin regions (output data) and from both color and texture features of the skin regions (input data). Results: The proposed skin detection method achieved a true positive rate of approximately $90 \%$ and a false positive rate of approximately $0.22 \%$. Furthermore, this study analyzes and compares the obtained results from the proposed skin detection with thresholdbased skin detector to show the level of robustness, using both color and texture features in the proposed skin detector. Conclusion: It was found that a skin detector based on both color and texture features can lead to an efficient and more reliable skin detection method compared with other state-ofthe-art threshold-based skin detectors. The proposed detector reduces the FP rate to $0.22 \%$ compared with a skin detector based on predefined color rules.
\end{abstract}

Key words: Skin detection, FIS, image texture features, RGB color model

\section{INTRODUCTION}

Skin detection is used in determining pixels related to human skin. It is an important technique in image processing and the most distinctive and widely used key technology in many applications, such as face detection (Kovac et al., 2003), face tracking (Dadgostar et al., 2005), human motion analysis (Gavrila, 1999) and naked image filters (Fleck et al., 1996). One of the major issues in using skin color in skin detection is the task of choosing a suitable color space. Color is a useful cue to extract skin pixels. Numerous color models are being used today, because color science is a broad field encompassing many areas of applications. The most common color space models are RGB, CMY and CMYK (Gonzalez and Woods, 2002); Hue, Saturation and Intensity (HIS) (Umbaugh, 1997; Singh et al., 2003); Hue, Saturation and Value (HSV) (Lin et al., 2003); normalized RGB (Chan et al., 1999; Vezhnevets et al., 2003) and YCbCr (Umbaugh, 1997; Lin et al., 2003). Many skin models have been developed based on colors (RGB) (Vezhnevets et al., 2003), but these approaches are not robust enough to handle different lighting conditions and complex backgrounds containing surfaces and objects with skin-like colors. Many researchers (Gasparini et al., 2005; Shirali-Shahreza et al., 2008) have used pixel-based algorithms as the main methods for skin detection. Nevertheless, few skin detection methods have been constructed based on a pixel and its neighbors (region) (Ruiz-Del-Solar and Verschae, 2004). Some researchers have used traditional techniques (Jones and Rehg, 2002 Ghouzali et al., 2008; Maskooki et al., 2009), while others used intelligence (Brown et al., 2001; Bhoyar and Kakde, 2010; Subramanian et al., 2008) to detect skin pixels.

Corresponding Author: Alaa Y. Taqa, Department of Computer System and Technology, University of Malaya, 50603, Kuala Lumpur, Malaysia 
Table 1: Several publication of skin detection

\begin{tabular}{|c|c|c|}
\hline Reference/Author/Year & Paper title & Approach \\
\hline Almohair et al. (2007) & Skin detection in luminance images using threshold technique & Threshold values based skin detection \\
\hline Jiang et al. (2007) & Skin detection using color, texture and space information & $\begin{array}{l}\text { Integrate color, texture and space } \\
\text { information. Using Gabor filter }\end{array}$ \\
\hline Abin et al. (2008) & $\begin{array}{l}\text { Skin segmentation based on cellular } \\
\text { Learning automata }\end{array}$ & $\begin{array}{l}\text { Combining color and texture information } \\
\text { of skin with cellular learning automata }\end{array}$ \\
\hline Ahmed et al. (2007) & $\begin{array}{l}\text { A robust fuzzy logic based approach for } \\
\text { skin detection in colored images }\end{array}$ & Fuzzy logic using different color model \\
\hline Al-Wadud and Chae (2008) & A skin detection approach based on color distance map & DM based on gray scale images \\
\hline Conci et al. (2008) & $\begin{array}{l}\text { Comparing color and texture-based } \\
\text { algorithms for human skin detection }\end{array}$ & $\begin{array}{l}\text { Skin detection based on texture feature } \\
\text { using different color spaces }\end{array}$ \\
\hline Ghouzali et al. (2008b) & $\begin{array}{l}\text { A skin detection algorithm based on discrete cosine } \\
\text { transform and generalized Gaussian density }\end{array}$ & $\begin{array}{l}\text { Skin model based on generalized Gaussian } \\
\text { density }\end{array}$ \\
\hline Fotouhi et al. (2009) & Skin detection using contourlet-based texture analysis & $\begin{array}{l}\text { Color and texture based on wavelet domain } \\
\text { using neural network }\end{array}$ \\
\hline Zafarifar et al. (2010) & $\begin{array}{l}\text { Improved skin segmentation for TV Image } \\
\text { enhancement, using color and texture features }\end{array}$ & $\begin{array}{l}\text { defined in HSV color space and versus a } \\
\text { histogram-based color detector and extract } \\
\text { texture feature }\end{array}$ \\
\hline
\end{tabular}

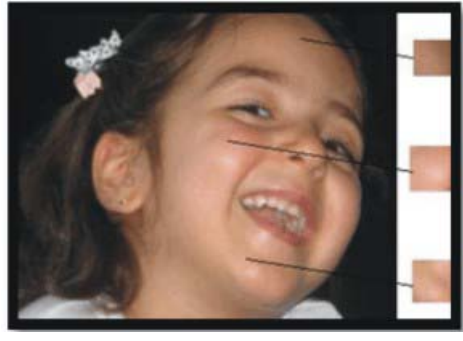

Fig. 1: Different light conditions

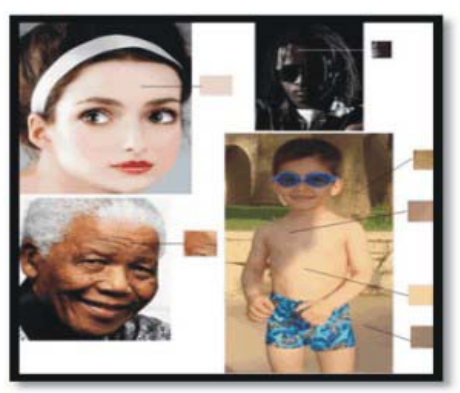

Fig. 2: Different skin and skin-like tones light conditions

Table 1 illustrates several publications using different approaches for skin detection. This study proposes a novel reliable Fuzzy Inference System (FIS) for skin detection, which combines both color and texture features.

Skin detection: It could be defined as the process of finding skin-colored pixels and regions in an image or a video. There are two main approaches for skin detection, namely pixel-based and region-based. In pixel-based methods, the features (e.g., color) are extracted from information coming from a pixel; in region-based methods, the features (e.g., texture) are extracted from information about a pixel and its neighbors. Skin detection algorithms aim to recognize skin pixels in an unconstrained input image. Skin color is considered as a useful and discriminating spatial feature for many applications, but it is not robust enough to deal with complex image environments. Skin tones range from dark (some Africans) to light white (Caucasians and some Europeans). In addition, both the light-changing conditions (Fig. 1) and the existence of objects with skin-like colors could cause some major difficulties.

Figure 2 shows the different skin-color tones and skin-color-like tones. To help overcome these problems, this study proposes a novel FIS for skin detection that combines both color and texture features.

\section{MATERIALS AND METHODS}

Statistical-based texture features: Three texture features were estimated using a statistical approach, which computes the different properties through three statistical measures: standard deviation, maximumminimum range and entropy. These features were extracted from each pixel and its neighbors. The standard deviation could be calculated using the following formula (Verzani, 2004):

$\mathrm{S}=\left(\frac{1}{\mathrm{n}} \sum_{\mathrm{i}=1}^{\mathrm{n}}\left(\mathrm{x}_{\mathrm{i}}-\overline{\mathrm{x}}\right)^{2}\right)^{\frac{1}{2}}$

Where:

$\overline{\mathrm{x}}=\frac{1}{\mathrm{n}} \sum_{\mathrm{i}=1}^{\mathrm{n}} \mathrm{x}_{\mathrm{i}}$ 
Meanwhile, the maximum-minimum range equals (maximum value of pixel-minimum value of pixel) of the n-by-n neighborhood around the corresponding pixel in input image I (Gonzalez and Woods, 2002). Finally, the entropy was estimated by using the formula (Gonzalez and Woods, 2002):

$$
\mathrm{E}=\sum_{\mathrm{i}=1}^{\mathrm{n}} \mathrm{P}\left(\mathrm{x}_{\mathrm{i}}\right) * \log _{2}\left(\mathrm{P}\left(\mathrm{x}_{\mathrm{i}}\right)\right)
$$

where, $\mathrm{P}\left(\mathrm{x}_{\mathrm{i}}\right)$ is the probability of the pixel color $\left(\mathrm{x}_{\mathrm{i}}\right)$ and $\mathrm{n}$ represents the number of pixels.

Generation of the fuzzy inference system: The term "fuzzy logic" emerged in the development of the theory of fuzzy sets by Zadeh (1965). Fuzzy logic provides a simple way of arriving at a definite decision based on vague, ambiguous, imprecise, noisy, or missing data. It generates an FIS structure from databases in order to generate a Sugeno-type fuzzy (Sugeno, 1985) inference system structure using subtractive clustering.

Subtractive clustering: Data clustering is considered as an interesting approach for determining similarities in data and categorizing similar data into groups (Visalakshi and Thangavel, 2009). The most representative off-line clustering techniques are illustrated in Table 2. Fuzzy clustering is an important class of clustering algorithms. It helps find natural vague boundaries in data $(\mathrm{Du}, 2010)$. The subtractive clustering method proposed by Yager and Filev (1994) is an example of fuzzy clustering. It is based on partitioning the data space into grids, with the density of each grid computed depending on the distance of the grid center to the data points.

A grid with many nearby data points will have a high potential value. The first cluster is selected based on the grid with the highest potential value to ensure that any two grids that are close together do not become two different clusters; the potential of the nearby grids is reduced based on their distance from the cluster center. The next cluster center is then selected from the remaining grids with the highest potential value.

Table 2: Example of clustering algorithms

\begin{tabular}{ll}
\hline Reference/Author/Year & Clustering algorithm \\
\hline MacQueen (1967) & K-means (or hard C-means) clustering \\
Johnson (1967) & hierarchical clustering \\
Dempster et al. (1977) & Mixture of Gaussian \\
Bezdek (1981) & The fuzzy cmeans algorithm \\
Kohonen (1982) & Kohonen's self-organizing map \\
Yager and Filev (1994) & Mountain method for clustering \\
Chiu (1994) & Subtractive clustering \\
\hline
\end{tabular}

Chiu (1994) further developed this idea by using actual data points as cluster centers, rather than grids. Each data point is given a potential value based on its neighboring points and the point with the highest potential value is considered the cluster center (Sampath and Shan, 2008). However, the subtractive clustering method assumes that each data point is a potential cluster center and calculates the likelihood that each data point would define the cluster center based on the density of surrounding data points. The data point with the highest potential value, which will be the first cluster center, is selected, followed by all other points. To determine the next data cluster and its center location, all data points near the first cluster center (as determined by the cluster center range) are then removed. This process is repeated until all data points are within the range of the cluster center. A data point with the highest potential, which is a function of the distance measure, is considered the cluster center. The potential of each data point is estimated using the following formula (Chiu, 1994; 1996):

Where:

$P_{i}=\sum_{i=1}^{n} e^{-x}\left\|x^{i}-x^{j}\right\|^{2}$

$\propto=4 / r_{a}^{2}$

Where:

$\mathrm{r}_{\mathrm{a}}=$ A positive constant

$\|\|=$. The Euclidean distance

Thus, to measure the potential value for a data point, the distance from this point to all other data points is computed. A data point with many neighboring data points will have a high potential value. The constant $r_{a}$ is the radius defining a neighborhood; data points outside this radius have little influence on the potential value. Once the potential value of each data point is computed and that with the highest potential value is selected as the first cluster center. Let $x^{*} 1$ be the location of the first cluster center and $\mathrm{P} * 1$ be its potential value. The potential value of each data point $\mathrm{x}^{\mathrm{i}}$ is then revised by the following formula (Chiu, 1994; 1996):

Where:

$$
\begin{aligned}
& \mathrm{P}_{\mathrm{i}} \Leftarrow \mathrm{P}_{\mathrm{i}}-\mathrm{P}_{1}^{*} \mathrm{e}^{-\beta}\left\|\mathrm{x}_{\mathrm{i}}-\mathrm{x}_{1}^{*}\right\|^{2} \\
& \beta=4 / \mathrm{r}_{\mathrm{b}}^{2}
\end{aligned}
$$

and $r_{b}$ is a positive constant. Thus, the amount of potential value is subtracted from each data point as a 
function of its distance from the first cluster center. Data points near the first cluster center will have greatly reduced potential value and will, therefore, be unlikely selected as the next cluster center. The constant $r_{b}$ is the radius defining the neighborhood, which will have measurable reductions in potential value. When the potential value of all data points has been revised, the data point with the highest remaining potential value is selected as the second cluster center. Afterwards, the potential value of each data point is reduced according to their distance to the second cluster center. In general, after the k'th cluster center is obtained, the potential of each data point is revised by the following formula (Chiu, 1994; 1996):

$\mathrm{P}_{\mathrm{i}} \Leftarrow \mathrm{P}_{\mathrm{i}}-\mathrm{P}_{\mathrm{k}}^{*} \mathrm{e}^{-\beta}\left\|\mathrm{x}_{\mathrm{i}}-\mathrm{x}_{\mathrm{k}}^{*}\right\|^{2}$

Where:

$\mathrm{x}_{\mathrm{k}}^{*}=$ The location of the $\mathrm{k}^{\prime}$ th cluster center

$\mathrm{P}_{\mathrm{k}}^{*}=$ Its potential value

This process is repeated until the remaining potential values of all data points fall below some fraction of the potential value of the first cluster center $\mathrm{P}_{1}^{*}$.

Sugeno-type fuzzy inference: The Sugeno or TakagiSugeno-Kang (Sugeno, 1985) FIS algorithm is similar to the Mamdani method (Mamdani and Assilian, 1975) in many aspects. The first two parts of the fuzzy inference process-fuzzifying the inputs and applying the fuzzy operator-are exactly the same. The main difference between Mamdani and Sugeno is that the Sugeno output membership functions are either linear or constant. A typical rule in a Sugeno fuzzy model has the following form (Mamdani and Assilian, 1975): If input $1=\mathrm{x}$ and input $2=y$, then output is $z=a x+b y+c$.

For a zero-order Sugeno model, the output level $\mathrm{z}$ is a constant $(a=b=0)$. The output level $z_{i}$ of each rule is weighted by the firing strength $\mathrm{w}_{\mathrm{i}}$ of the rule. For example, for an AND rule with input $1=\mathrm{x}$ and input 2 $=\mathrm{y}$, the firing strength is:

$$
\mathrm{W}=\text { And Method (F1(x), F2(y)) }
$$

where, F1 and F2 are the membership functions for inputs 1 and 2, respectively.

The final output of the system is the weighted average of all rule outputs, which is computed as (Tang et al., 2007):

$$
\text { Finaloutput }=\frac{\sum_{\mathrm{i}=1}^{\mathrm{N}} \mathrm{w}_{\mathrm{i}} \mathrm{z}_{\mathrm{i}}}{\sum_{\mathrm{i}=1}^{\mathrm{N}} \mathrm{w}_{\mathrm{i}}}
$$

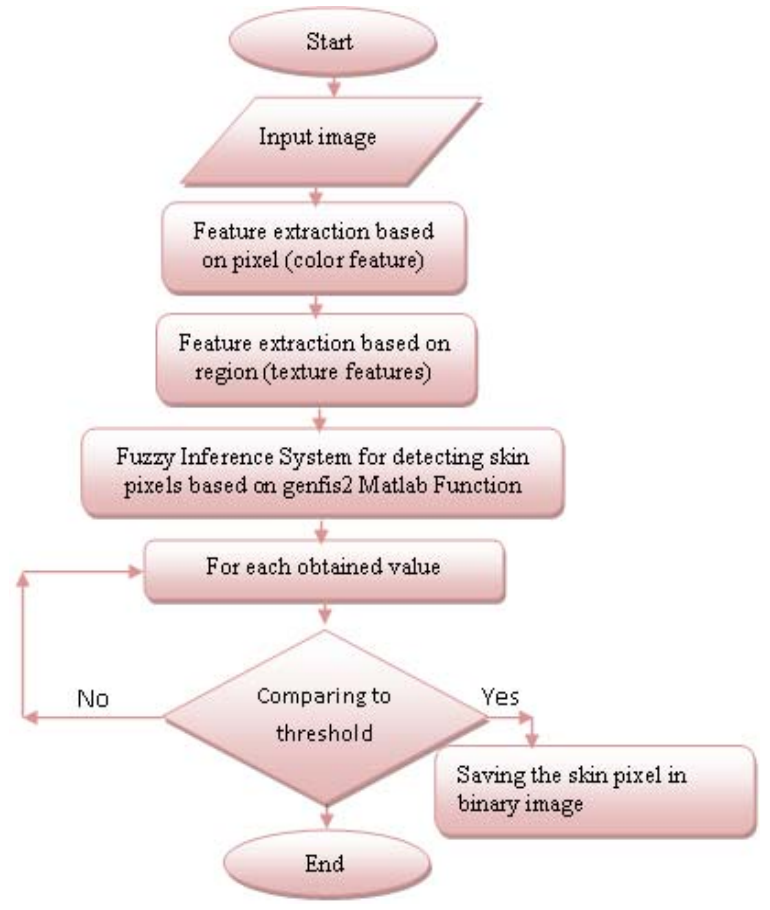

Fig. 3: The prposed fos based skin detection

The proposed skin detection algorithms: The proposed FIS based on Sugeno reasoning for skin detection combines both color and texture features. To increase the reliability of the skin detection process, neighborhood pixel information is incorporated into the proposed method. The color features are extracted directly from the pixels and the texture features of the scanned windows over the image are extracted using a statistical approach to produce feature vector. To determine the decision rule of these features, an FIS is used. The overall structure of the proposed skin detection is illustrated in Fig. 3.

Data set: The creation of skin and non-skin image database involved collecting samples of different human skin-colored pixels from a variety of people under different illumination conditions (skin pixels without background), as well as a variety of non-skincolored pixels. An image was examined manually to determine whether it contained skin. If no skin was present, the image was placed in the non-skin group. In the skin image group, regions of skin pixels were manually extracted using Adobe Photoshop. In labeling skin, the eyes, hair, clothes, mouth opening and lips were all excluded. The collected data were then divided into two subsets: constructing ("constructing_set") and testing ("testing_set"). The constructing_set was used as the primary set of data applied in constructing FIS, 
with 351,228 skin pixels and 428,602 non-skin pixels. The test set included different images with simple and complex backgrounds, indoor and outdoor settings and different image sizes and skin colors used to measure the performance of the proposed skin detection. It had 632,379 different pixel types.

Feature extraction: Three statistical measures were used to estimate the texture features-standard deviation, maximum-minimum range and entropy. These features were extracted from each pixel and its neighbors and two windows were moved over an image; the size of the first window was $3 \times 3$ and that of the second was $9 \times 9$. The color features (red, green and blue) of the center pixel of the first window were extracted and the first two static features were then estimated from pixels within the first window. Afterwards, entropy was estimated from the pixels within the second window. These six features were used as inputs to the fuzzy inference system. All statistical measures were computed for multi-channel image matrices (red, green and blue) and their average was determined. Figure 4 shows an example of the scheme of computing entropy for multi-channel image matrices.

Entropy is a statistical measure of randomness and is used to characterize the texture of the input image (Gonzalez and Woods, 2002). Entropy is defined for red, green and blue channels, respectively, as:

$$
\begin{aligned}
& \mathrm{E}_{\mathrm{r}}=\sum_{\mathrm{i}=1}^{\mathrm{n}} \mathrm{P}_{\mathrm{r}}\left(\mathrm{x}_{\mathrm{i}}\right) * \log _{2}\left(\mathrm{P}_{\mathrm{r}}\left(\mathrm{x}_{\mathrm{i}}\right)\right) \\
& \mathrm{E}_{\mathrm{g}}=\sum_{\mathrm{i}=1}^{\mathrm{n}} \mathrm{P}_{\mathrm{g}}\left(\mathrm{x}_{\mathrm{i}}\right) * \log _{2}\left(\mathrm{P}_{\mathrm{g}}\left(\mathrm{x}_{\mathrm{i}}\right)\right) \\
& \mathrm{E}_{\mathrm{b}}=\sum_{\mathrm{i}=1}^{\mathrm{n}} \mathrm{P}_{\mathrm{b}}\left(\mathrm{x}_{\mathrm{i}}\right) * \log _{2}\left(\mathrm{P}_{\mathrm{b}}\left(\mathrm{x}_{\mathrm{i}}\right)\right)
\end{aligned}
$$

The average entropy matrix will be:

$$
E=\frac{E_{r}+E_{g}+E_{b}}{3}
$$

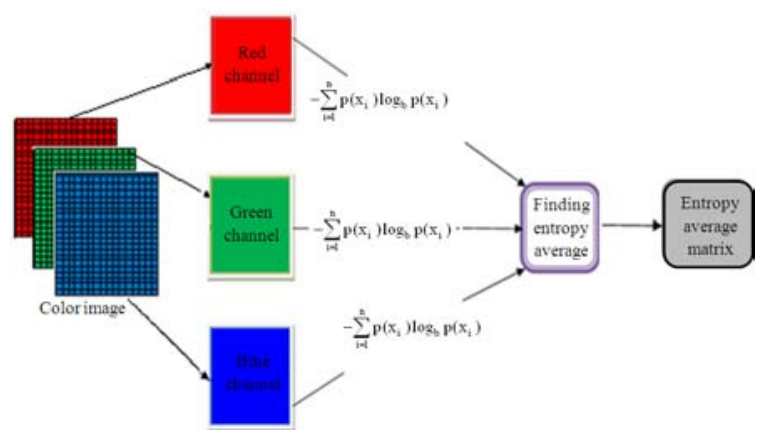

Fig. 4: Computing scheme entropy for colored image
Fuzzy inference system: The FIS mainly consists of two phases-the construction phase and the test phase. The first two steps of both phases are inputting image and extracting features from such an image.

Construction phase: The FIS was constructed using a priori knowledge about skin and non-skin pixels that were extracted from certain images (construction_set) in order to detect skin pixels using the genfis2 Matlab function. The rule extraction method is based on estimating clusters in the data; each cluster obtained corresponds to a fuzzy rule that relates a region in the input space to suitable output region. The construction phase generates a Sugeno-type FIS structure using both subtractive clustering algorithm and separate sets of input and output data. A set of rules that models the data behavior was extracted using subtractive clustering algorithm. Throughout this phase, the FIS structure containing a set of fuzzy rules to cover the feature space (constrution_set) was obtained. Figure 5 illustrates the construction phase of the FIS.

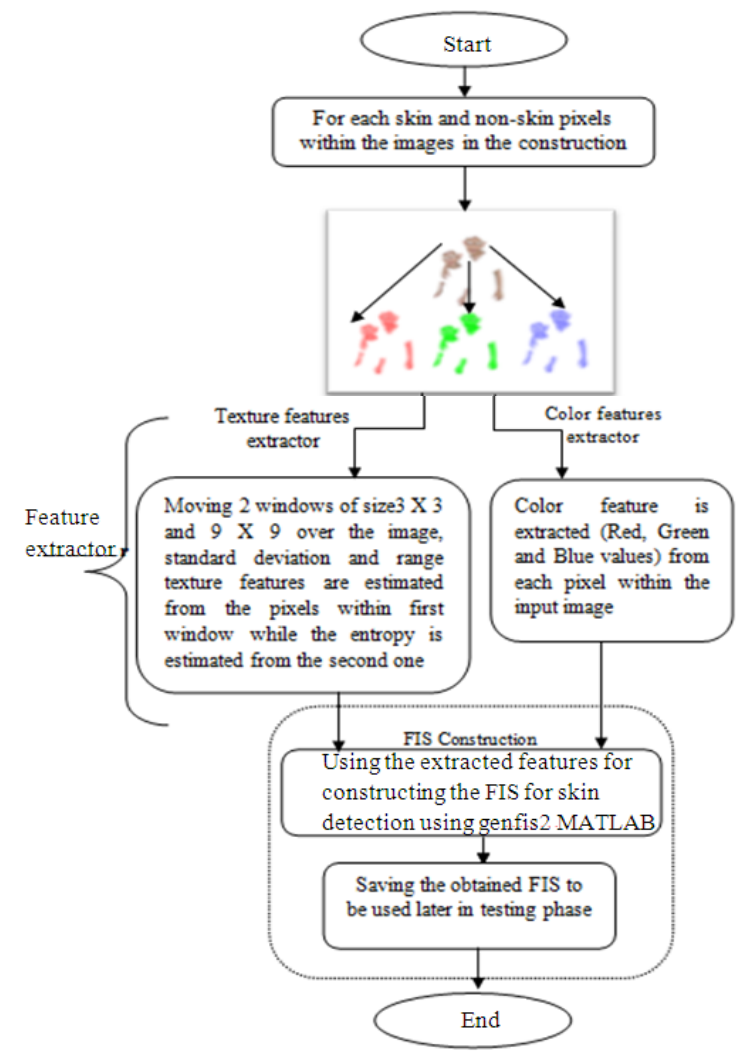

Fig. 5: Constructing phase of the proposed skin detection 


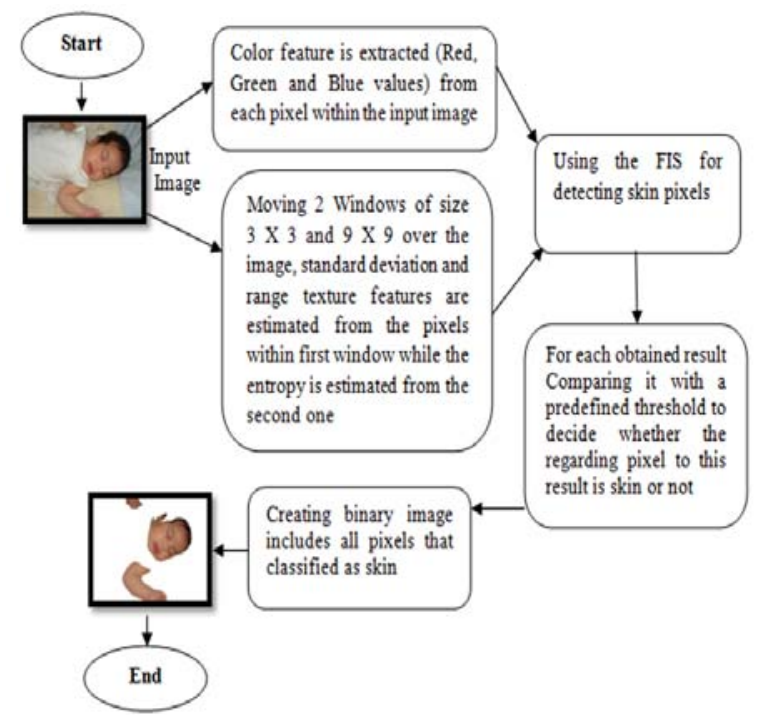

Fig. 6: Testing phase of the proposed skin detection

Testing phase: A skin detector was used to test each pixel of a given image (test image) depending on the FIS. If a pixel was detected as skin, then it was stored in a new image (skin image) at the position of the original image. After examining all image pixels, a new binary image was obtained, including only skin pixels, as shown in Fig. 6.

\section{RESULTS}

The FIS was constructed based on the genfis2 Mathlab system. The main structure of the FIS is shown in Fig. 7. It has six inputs, each representing a single feature (SD, Entropy, Range, Red, Green and Blue) and one output, either skin or non-skin pixels.

The road map of the proposed FIS for skin detection is shown in Fig. 8. It represents the rules of the system. A single-figure window with 36 plots nested in it. The six plots across the top of the Fig. 8 represent the antecedent and consequent of the first rule. Each rule is a row of plots and each column is a variable. The rule numbers are displayed on the left of each row. The first six columns of plots show the membership functions referenced by the antecedent or the if-part of each rule. The last column of plots shows the membership functions referenced by the consequent or the then-part of each rule. The sixth plot in the last column of plots represents the aggregate weighted decision for the given inference system.

The implementation of the skin detector was conducted by testing different images with simple and complex backgrounds, indoor and outdoor settings, as well as different image sizes and skin colors.

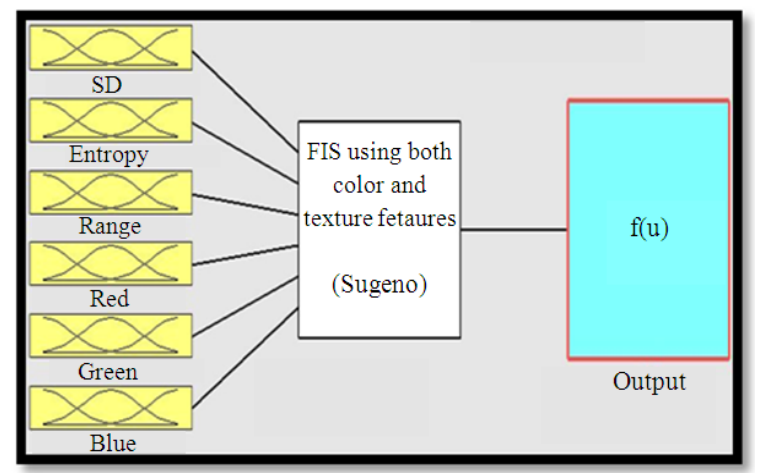

Fig. 7: The main structure of FIS

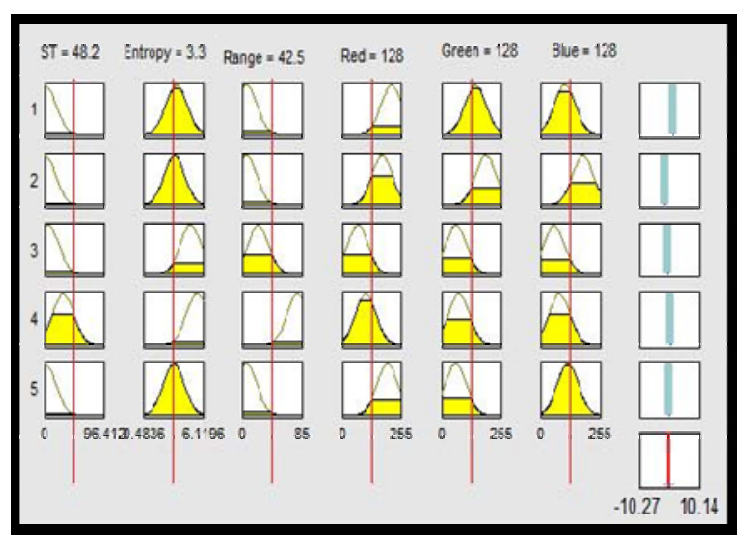

Fig. 8: The rules of FIS

An experiment was performed on the testing set, which included 632,379 uncontrolled (different illumination, captured quality, distance to camera) pixels. Of these, each of the first 92,883 pixels belonged to an arbitrary number of skin images and images containing an arbitrary number of people and faces and the remaining 539,496 pixels reflected no skin pixels and included pixels belonging to images with objects presenting skin-like tones (e.g., red flower, dog, chocolate).

Two different skin detectors were tested and evaluated to select the one with higher reliability; afterwards, the evaluations were compared with the performance of previous skin detectors. The first skin detection method detects skin pixels based on the threshold skin color tones (Kovac et al., 2003; Gasparini and Schettini, 2006), while the second detects skin pixels using the proposed skin detector, which combines both texture and color features.

The original images shown in Fig. 9 are segmented based on predefined color rules. The obtained images show that many non-skin pixels are detected incorrectly as skin pixels. On the other hand, there are no skin pixels detected in the last set of images. 


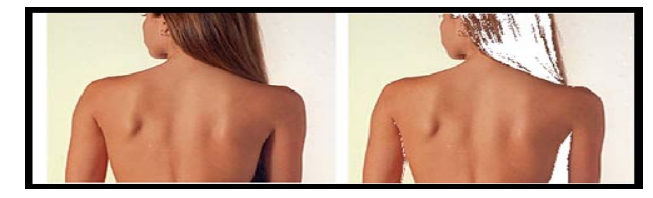

Original image Threshold based skin detector

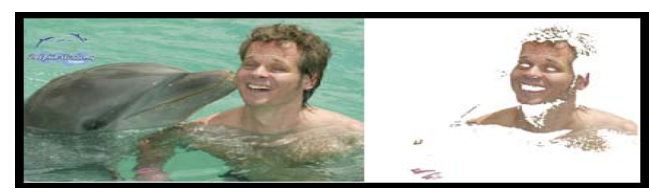

Original image

Threshold based skin detector

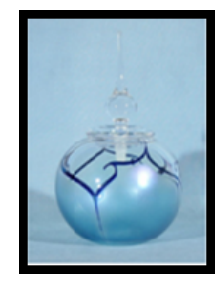

Original image

Fig. 9: Testing results using predefined color rules based skin detection
The testing results of the proposed FIS-based skin detector using different threshold values are illustrated in Fig. 10. The results include high rates of true positives and true negatives with low rates of false detection. Although images 1, 2, 3 and 4 in Fig. 10 reflect several human skin types with different colors and textures, the skin pixels within these images were detected correctly by the proposed skin detector, except for a few scattered pixels that were incorrectly detected as non-skin pixels. Most skin pixels within the images shown in Fig. 10 were detected correctly; meanwhile, no false detection rates were shown within image 5 for a threshold value greater than 0.5 and within image 6 for all threshold values.

A skin detection process is not perfect and different users adopt varying criteria for performance evaluation. One of the evaluation criteria consists of the general appearance of the size zones detected. To quantify performance evaluation, True Positive (TP), False Positive (FP), True Negative (TN) and False Negative (FN) were computed for all pixels in the testing_set through skin detector testing. FP is the proportion of nonskin pixels classified incorrectly as skin, whereas TP is the proportion of skin pixels classified correctly as skin.

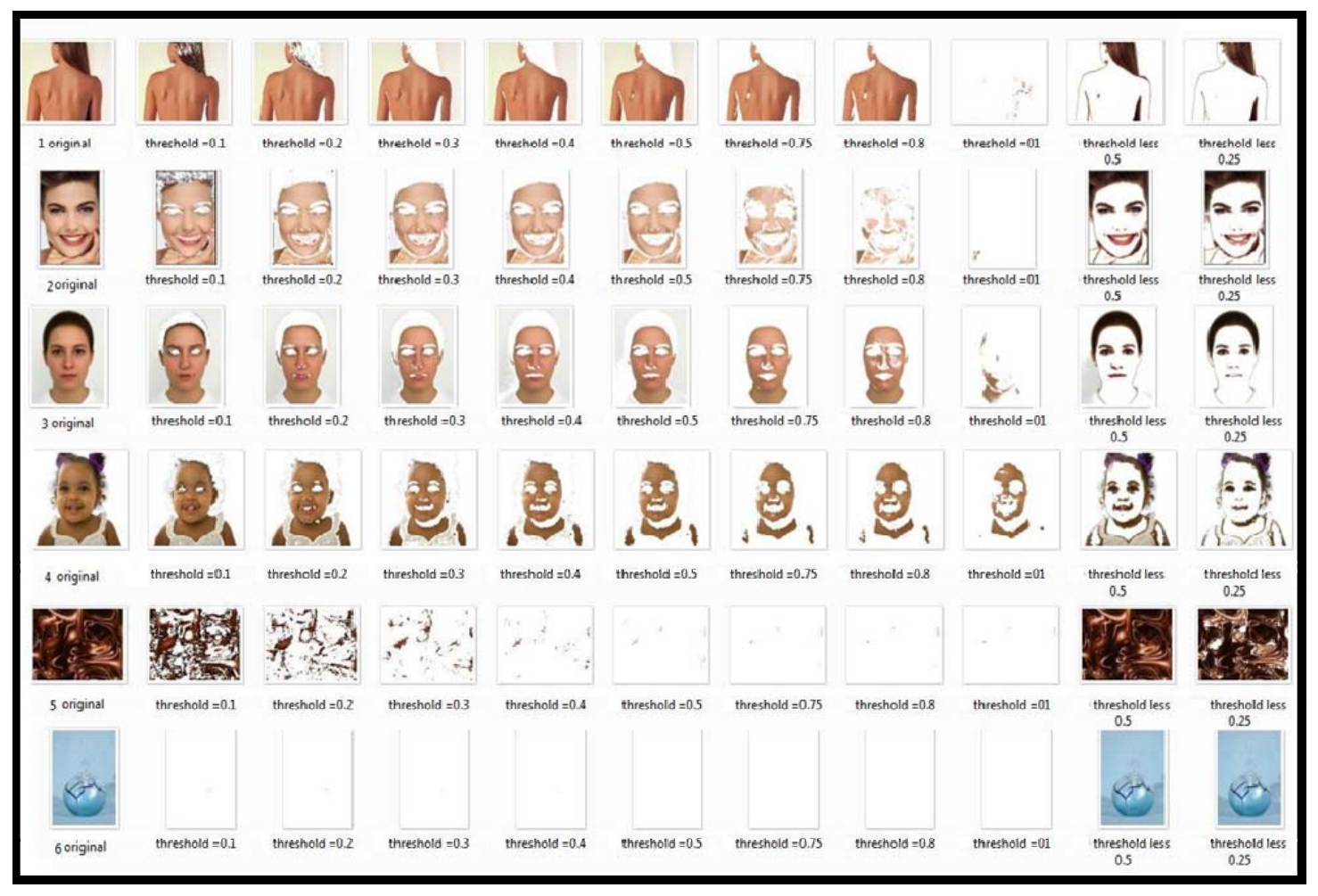

Fig. 10: Testing results using the proposed FIS based skin detection 
Am. J. Applied Sci., 7 (8): 1129-1138, 2010

Table 3: True positive and false positive of skin detections

\begin{tabular}{|c|c|c|c|c|}
\hline \multicolumn{2}{|l|}{ Skin detector } & \multicolumn{2}{|c|}{$\mathrm{TP}(\%)$} & FP $(\%)$ \\
\hline \multirow{2}{*}{\multicolumn{2}{|c|}{$\begin{array}{l}\text { Threshold based skin detector } \\
\text { FIS based Skin detector using } \\
\text { both texture and color features }\end{array}$}} & \multirow{2}{*}{\multicolumn{2}{|c|}{$\begin{array}{l}84.98 \\
90.00\end{array}$}} & 8.45 \\
\hline & & & & 0.22 \\
\hline \multicolumn{5}{|c|}{ Table 4: Evaluation metrics of skin detections } \\
\hline Skin detector & Recall & Precision & Specificity & Accuracy \\
\hline Threshold based Skin detector & 0.828 & 0.675 & 0.804 & 0.871 \\
\hline $\begin{array}{l}\text { FIS based Skin detector using } \\
\text { both texture and color features }\end{array}$ & 0.900 & 0.700 & 0.910 & 0.910 \\
\hline
\end{tabular}

TN and FN are the complements of FP and TP, respectively. To evaluate the proposed skin detector, a sample group consisting of 632,379 pixels was used in this evaluation (Table 3 ).

Four metrics (Table 4) were used to evaluate the performance of the two skin detections. These metrics are: recall, precision, specificity and accuracy (Gasparini et al., 2005; Fawcett, 2004).

\section{DISCUSSION}

Considering the unconstrained nature of internet images, the performance of the proposed skin detector is surprisingly good. The best performance can detect $90 \%$ of skin pixels with a very low FP rate of $0.22 \%$ by combining both texture and color features. Although the threshold based skin detection method can detect $84.98 \%$ of skin pixels correctly, it also has a high FP rate of $8.45 \%$. A simple comparison among the performance evaluation of the two skin detection methods is shown in Fig. 11.

Although there is no means to locate any two papers using the same test sets, examining previously published results may be useful. The performance of the proposed skin detector in this research is thus compared with that of other skin detectors. The Bethe tree approximation of first order model proposed by (Abdullah-Al-Wadud and Chae, 2008) can detect 72\% of skin pixels with a $5 \%$ FP rate, whereas the proposed Bayesian model by (Jones and Rehg, 2002) can detect $69 \%$ at the same FP rate. Meanwhile, this model can detect $80 \%$ of skin pixels with an $8.5 \%$ FP rate or $90 \%$ correct detection with $14.2 \%$ FP. The skin detection method suggested by Zafarifar et al. (2010) can detect more than $83 \%$ of skin pixels correctly with a $20 \%$ FP rate. The recall rate of the pixel-based skin color classification proposed by (Gasparini et al., 2005) is $92 \%$ and the precision rate is $39 \%$. These evaluation metric values indicate that the proposed skin detector outperforms other methods mentioned above, especially in terms of decreasing the FP rate.

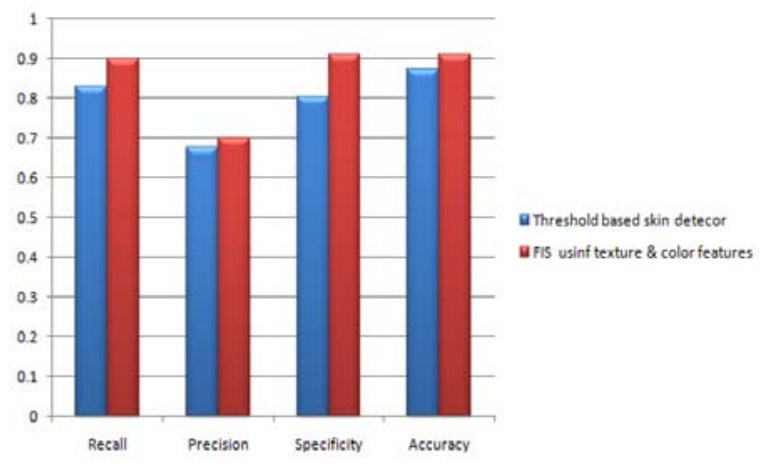

Fig. 11: Evaluation comparisons between two skin detections

\section{CONCLUSION}

Skin detection is an important pre-processing stage in many image analysis applications; hence, we proposed an improved FIS for skin detection, which combines both color and texture features to increase the reliability of the proposed method. Neighborhood information of each pixel was also used throughout the training and testing phases. Two skin detections (threshold-based skin detector and a combination of both color and texture features) were applied and tested. This study demonstrated that a skin detector based on both color and texture features can lead to an efficient and more reliable skin detection method compared with that based on threshold. The proposed detector reduces the FP rate to $0.22 \%$ compared with a threshold based skin detector. An essential future direction will be to validate the proposed algorithms using a standard skin database data set. Such a method will enable us to compare our detection results with those presented by other authors for the same test images. Another improvement would be to adapt our approach in wavelet domain.

\section{REFERENCES}

Abdullah-Al-Wadud, M. and O. Chae, 2008. A skin detection approach based on color distance map. EURASIP J. Adv. Sign. Process., 2008: 1-10. DOI: $10.1155 / 2008 / 814283$

Abin, A.A., M. Fotouhi and S. Kasaei, 2008. Skin segmentation based on cellular learning automata. Proceeding of the 6th International Conference on Advances in Mobile Computing and Multimedia, Nov. 24-26, ACM Press, Linz, Austuria, pp: 254-259. DOI: $10.1145 / 1497185.1497238$ 
Ahmed, A., M.Y. Javed and U. Qayyum, 2007. A robust fuzzy logic based approach for skin detection in colored images. Proceeding of the 11th International Conference on Artificial Intelligence and Soft Computing, Aug. 29-31, ACM Press, Palma de Mallorca, Spain, Anaheim, CA., USA., pp: 295-301.

http://portal.acm.org/citation.cfm?id=1659652.1659707

Almohair, H.K., A.R. Ramli, A.M. Elsadig and S.J. Hashim, 2007. Skin detection in luminance images using threshold technique. Proc. Int. J. Comput., Internet Manage., 15: 25-32. http://www.journal.au.edu/ijcim/2007/jan07/IJCIM vol15no1 article3.pdf

Bezdek, J.C., 1981. Pattern Recognition with Fuzzy Objective Function Algorithms. 1st Edn., Springer, New York, ISBN: 10: 0306406713, pp: 272.

Bhoyar, K.K. and O.G. Kakde, 2010. Skin color detection model using neural networks and its performance evaluation. J. Comput. Sci., 6: 955-960. http://www.scipub.org/fulltext/jcs/jcs69955-960.pdf

Brown, D.A., I. Craw and J. Lewthwaite, 2001. A SOM based approach to skin detection with application in real time systems. Proceeding of the British Machine Vision Conference, Sept. 10-13, Manchester, UK., pp: 491-499. http://www.comp.leeds.ac.uk/bmvc2008/proceedin gs/2001/papers/33/accepted_33.pdf

Chan, Y., R. Harvey and D. Smith, 1999. Building systems to block pornography. Proceeding of the 2nd UK Conference on Image Retrieval: The Challenge of Image Retrieval, BCSEI Electronic Workshops in Computing Series, Feb. 25-26, Citeseerx, New Castle, UK., pp: 34-40. http://citeseerx.ist.psu.edu/viewdoc/summary?doi= 10.1.1.13.25

Chiu, S.L., 1994. Fuzzy model identification based on cluster estimation. J. Intell. Fuzzy Syst., 2: 267-278. http://chius.homestead.com/files/fuzzymodelid.pdf

Chiu, S.L., 1996. Extracting Fuzzy Rules from Data for Function Approximation and Pattern Classification. In: Fuzzy Information Engineering: A Guided Tour of Application, Dubois, D., H. Prade and R. Yager (Eds.). Wiley, USA., ISBN: 10: 0471147664, pp: 149-162.

Conci, A., E. Nunes, J.J. Pantrigo and A. Sanchez, 2008. Comparing color and texture-based algorithms for human skin detection. Proceeding of the 10th International Conference on Enterprise Information Systems, June 12-16, HCI, Barcelona, Spain, pp: 166-173. http://www.gavab.etsii.urjc.es/blosxom/publicacion es_new/ano2008/iceis_paper268_v2.pdf
Dadgostar, F., A. Sarrafzadeh and S.P. Overmyer, 2005. Face tracking using mean-shift algorithm: A fuzzy approach for boundary detection. Lecture Notes Comput. Sci., 3784: 56-63. DOI: 10.1007/11573548_8

Dempster, A.P., N.M. Laird and D.B. Rubin, 1977. Maximum likelihood from incomplete data via the EM algorithm. J. R. Stat. Soc., Ser. B, 39: 1-38. http://www.citeulike.org/user/ykuoka/article/117535

Du, K.L., 2010. Clustering: A neural network approach. Neural Networks, 23: 89-107. DOI: 10.1016/j.neunet.2009.08.007

Fawcett, T., 2004. ROC graphs: Notes and practical consideration for researchers. Kluwer Academic Publishers.

http://home.comcast.net/ tom.fawcett/public_html/ papers/ROC101.pdf

Fleck, M.M., D. Forsyth and C. Bregler, 1996. Finding naked people. Lecture Notes Comput. Sci., 1065: 593-602. DOI: 10.1007/3-540-61123-1_173

Fotouhi, M., M.H. Rohban and S. Kasaei, 2009. Skin detection using contourlet texture analysis. Proceeding of the 4the International Conference on Digital Telecommunications, July 20-25, IEEE Xplore Press, Colmar, pp: 59-64. DOI: 10.1109/ICDT.2009.18

Gasparini, F. and R. Schettini, 2006. Skin segmentation using multiple thresholding. Proc. SPIE Int. Soc. Optic. Eng., 6061: 60610F.1-60610F. http://cat.inist.fr/?aModele=afficheN\&cpsidt=18971809

Gasparini, F., S. Corchs, and R. Schettini, 2005. Pixel based skin color classification exploiting explicit skin cluster definition methods. Proceeding of the AIC Color Congress of the International Color Association, May 5-10, IVL, Granda, Spain, pp: 543-546.

http://www.ivl.disco.unimib.it/papers2003/aic2005 $-543 \_546 . p d f$

Gavrila, D.M., 1999. The analysis of human motion and its application for visual surveillance. Proceeding of the 2nd IEEE Workshop on Visual Surveillance, June 26-26, IEEE Xplore Press, Fort Collins, CO., USA., pp: 3-5. DOI: 10.1109/VS.1999.780260

Ghouzali, S., S. Hemami, M. Rziza, D. Aboutajdine and E.M. Mouaddib, 2008. A skin detection algorithm based on discrete cosine transform and generalized Gaussian density. Proceeding of the 15th IEEE International Conference on Image Processing, Oct. 12-15, IEEE Xplore Press, San Diego, CA., pp: 605-608. DOI: 10.1109/ICIP.2008.4711827

Gonzalez, R.C. and R.E. Woods, 2002. Digital Image Processing. 2nd Edn., Prentice Hall, Inc., USA., ISBN: 0201180758, pp: 793. 
Jiang, Z., M. Yao and W. Jiang, 2007. Skin detection using color, texture and space information. Proceeding of the 4rth International Conference on Fuzzy Systems and Knowledge Discovery, Aug. 24-27, IEEE Xplore Press, Haikou, pp: 366-370. DOI: $10.1109 /$ FSKD.2007.518

Johnson, S.C., 1967. Hierarchical clustering schemes. Psychometrika, 32: 241-254. DOI: 10.1007/BF02289588

Jones, M.J. and J.M. Rehg, 2002. Statistical color models with application to skin detecting. Int. J. Comput. Vis., 46: 81-86. DOI: 10.1023/A:1013200319198

Kohonen, T., 1982. Self-organized formation of topologically correct feature maps. Biol. Cybernet., 43: 59-69. DOI: 10.1007/BF00337288

Kovac, J., P. Peer and F. Solina, 2003. Human skin color clustering for face detection. Proceeding of the International Conference on Computer as a Tool, Sept. 22-24, IEEE Xplore Press, USA., pp: 144-148. DOI: 10.1109/EURCON.2003.1248169

Lin, Y.C., H.W. Tseng and C.S. Fuh, 2003. Pornography detection using support vector machine. Proceeding of the 16th IPPR Conference on Computer Vision, Graphics and Image Processing, Aug. 17-19, Kinmen, ROC, pp: 123-130. http://www.csie.ntu.edu.tw/ fuh/personal/Pornogra phyDetectionUsingSupportVectorMachine.pdf

MacQueen, J., 1967. Some methods for classification and analysis of multivariate observations. Proceeding of the of 5th Berkeley Symposium on Mathematical Statistics and Probability, (MSP'67), University of California Press, Berkeley, pp: 281-297. http://projecteuclid.org/euclid.bsmsp/1200512992

Mamdani, E.H. and S. Assilian, 1975. An experiment in linguistic synthesis with a fuzzy logic controller. Inter. J. Man-Mach. Stud., 7: 1-13. DOI: 10.1016/S0020-7373(75)80002-2

Maskooki, A., E. Gunawan, C.B. Soh and K.S. Low, 2009. Frequency domain skin artifact removal method for ultra-wideband breast cancer detection. Prog. Electromag. Res., 98: 299-314. http://www.jpier.org/PIER/pier.php?paper=09101302

Ruiz-Del-Solar, J. and R. Verschae, 2004. Robust skin segmentation using neighborhood information. Proceeding of the IEEE International Conference on Image Processing, Oct. 24-27, IEEE Xplore Press, USA.,pp: 207-210. DOI: 10.1109/ICIP.2004.1418726

Sampath, A. and J. Shan, 2008. Building roof segmentation and reconstruction from LiDAR point clouds using clustering techniques. Int. Arch. Photogramm. Remote Sens. Spat. Inform. Sci., 37: 279-284.
Shirali-Shahreza, S.S., H. Beigy, M.H. ShiraliShahreza, 2008. Knapsack model for pixel based skin detection. Proceeding of the 4th IEEE International Conference on Intelligent Information Hiding and Multimedia Signal Processing, Aug. 15-17, IEEE Xplore Press, Harbin, pp: 581-584. DOI: 10.1109/IIH-MSP.2008.92

Singh, S.K., D.S. Chauhan, M. Vatsa and R. Singh, 2003. A robust skin color based face detection algorithm. Tamkang J. Sci. Eng., 6: 227-234. http://citeseerx.ist.psu.edu/viewdoc/summary?doi= 10.1.1.63.2881

Subramanian, S., K. Kumar B.P. Mishra, A. Banerjee and D. Bhattacharya, 2008. Fuzzy logic based content protection for image resizing by seam carving. Proceeding of the IEEE conference in Soft Computing in Industrial Applications, June 25-27, IEEE Xplore Press, Muroran, pp: 78-83. DOI: 10.1109/SMCIA.2008.5045939

Sugeno, M., 1985. Industrial Applications of Fuzzy Control. 1st Edn., Elsevier Science Ltd., New York, USA., ISBN: 10: 0444878297, pp: 278.

Tang, N., Y.H. Sun and Z. Chen, 2007. Multiple models switching method based on Sugeno fuzzy inference. Proceeding of the 6th International Conference on Machine Learning and Cybernetics, Aug. 19-22, IEEE Xplore Press, Hong Kong, pp: 1283-1287. DOI: 10.1109/ICMLC.2007.4370342

Umbaugh, S.E., 1997. Computer Vision and Image Processing: A Practical Approach Using CVIPTools. Prentice Hall, USA., ISBN: 10: 0132645998, pp: 528.

Verzani, J., 2004. Using R for Introductory Statistics. 1st Edn., Chapman and Hall/CRC, Boca Raton, FL., ISBN: 10: 1584884509, pp: 432.

Vezhnevets, V., V. Sazonov and A. Andreeva, 2003. A survey on pixel-based skin color detection techniques. Proceeding of the Graphicon-2003, (GRAPHICON'03), Springer, USA., pp: 85-92.

Visalakshi, N.K. and K. Thangavel, 2009. Distributed data clustering: A comparative analysis. Stud. Computat. Intell., 206: 371-397. DOI: 10.1007/978-3-642-01091-0

Yager, R. and D. Filev, 1994. Generation of fuzzy rules by mountain clustering. J. Intell. Fuzzy Syst., 2: 209-219.

Zafarifar, B., A. Martinière and P.H.N. de With, 2010. Improved skin segmentation for TV image enhancement, using color and texture features. Proceeding of the IEEE International Conference on Consumer Electronics, Jan. 9-13, IEEE Xplore Press, Las Vegas, USA., pp: 373-374. DOI: 10.1109/ICCE.2010.5418755

Zadeh, L.A., 1965. Fuzzy sets. Inform. Control, 8: 338-353. DOI: 10.1016/S0019-9958(65)90241-X 